\title{
An Integrated Approach for QoS-Aware Multicast Tree Maintenance
}

\author{
Wu-Hong Tsai ${ }^{1}$ and Yuan-Sun $\mathrm{Chu}^{2}$ \\ 1 Transworld Institute of Technology, Yunlin, 640 Taiwan \\ edward@tit.edu.tw \\ ${ }^{2}$ Chung Cheng University, Chiayi, 621 Taiwan \\ chu@ee.ccu.edu.tw
}

\begin{abstract}
To maintain the quality of multicast tree in supporting dynamic membership is critical important for the tree receivers. We propose an integrated approach that combines core migration with local search to afford the receivers a consistent quality service. The main ideas behind the thought are always migrate the core to the tree topological center within limited overhead and provide multiple routes via local search for a new receiver to meet its quality requirements. Our algorithm aims to meet a predefined upper bound delay along path to individual receiver and satisfy the constraint of inter-destination delay variation among all receivers. Simulation results show that our proposed algorithm performs well in terms of constrains indicated above.
\end{abstract}

Keywords: integrated approach; core migration; receiver; multicast tree.

\section{Introduction}

Multicast communication sends same messages to multiple destinations using shared links and efficiently uses resources such as bandwidth or buffer space. A multi-point connection adopted by multicast communication is a virtual topology which is usually a tree, called multicast tree. To construct a multicast tree in effectively supporting multimedia application such as video-conference or distancelearning has been widely researched. In particular, the research question of how to build a multicast tree to minimize the total cost of multi-type data transmitting over network is critical important. The total cost is the aggregated cost of all edges in the multicast tree that regards to tree performance. Multicast tree are not expected to only provide efficient usage of network resource, but also support quality requirements, in terms of real-time delay bound and a bound on the variation among the delays. For example, the participants in the videoconference want that all video frames are received smoothly (delay jitter) and simultaneously (inter-destination delay variation).

The tree shape is an important factor for delivering quality service and is affected by the category of receiver groups. Based on the nature of group membership, multicast receiver groups are classified into two categories: static group and dynamic group. With static group, the tree can be constructed in advance 
to fulfill quality requirements for the group. Yet, the overall information about network topology and the group size is required prior to tree construction. For dynamic group, the tree is dynamically constructed as members join/leave the group. Due to member's movement, the service qualities are often degraded, even though the tree is suitable for the group at first.

Two types of multicast trees, named source-based tree and core-based tree, have been considered to accommodate to quality requirements. In source-based tree, as a member requests for receiving a specific source's messages, it has to graft a shortest path onto the multicast tree that rooted at the specific source. And as a member joins multiple sources the multiple trees with shortest path are grafted as well. The more the resource of nodes is consumed the more the routing table of nodes is complicated. Instead of grafting onto multiple trees, a core node and a single shared tree, defined to be the union of the member-tocore shortest paths, is utilized in core-based tree (CBT) 1]. In CBT, messages destined for the group are first delivered to the core node, from which they are distributed along tree branches to group members. The shared tree in CBT reduces usage of resource and complications of routing table. Unfortunately, in CBT, the core location/selection problem becomes another issue which affects the tree shape and service quality requirements. Our research aims to provide a heuristic solution in resolving this important issue.

The rest of this paper is organized as follows. In Section 2, several related researches are reviewed. In section 3 , we define our research and propose a core selection/migration method that minimizes inter-destination delay variation of multicast tree. Simulation results are presented in Section 4 and some concluding remarks are given in Section 5 .

\section{Background}

The main issues associated with shared tree construction are core selection, paths setup for new members, and tree maintenance for core migration and member dynamics. The core location of a shared tree influences the performances of the tree in terms of total tree cost and delays experienced by individual receivers. The optimal core selection is an NP-complete problem and numbers of heuristics have been proposed in previous researches. Whereas most of these proposed heuristics require a completed topology and detailed membership exacted prior to core selection, and without taking the quality of services (QoS) requirements into account [2].

Ideally, while a new member requests to join a multicast group, multicast routing protocols should have the ability to find a feasible branch that connect the new member to the available multicast tree if it exists. CBT [1] grafts a new member to the multicast tree along the unicast routing path from core to the new member. It is suitable for best-effort traffic; however, in addition to consider QoS requirements, such shortest-path tree would not be acceptable. A few QoS-aware multicast routing protocols that use multiple branches searching method have been proposed in Carlberg's and Crowcroft's research [3. In their study, they mainly propose multiple candidate paths for new member to join the existed 
tree; and use GREEDY 4] approach in handling the leaving member. Where GREEDY [4] is, if the node being removed is a leaf node, then the branch of the tree supporting only that node is pruned; and for the case of non-leaf nodes, no action is taken.

However, in dynamic multicasting, continuous grafting and pruning degrades the performance of multicast tree over the time [2]. And the problem of updating a multicast tree to accommodate addition or deletion of nodes for the multicast group can be modeled as the on-line Steiner Problem. The on-line Steiner Problem in network is first presented by 4 and is an NP-complete problem as well. In the extreme case, the problem can be solved as a sequence of static multicast problem by rebuilding the tree at each stage using a static Steiner heuristic. However, this approach is too expensive in the cost and is unsuitable for ongoing real-time multicast sessions which cannot tolerate the disturbance caused by excessive change in the multicast tree after each addition or deletion.

Rouskas and Baldine [5] propose a source-based tree to meet the end-to-end delay constraints and inter-destination delay variation constraints by extension of path lengths. Wang et al. 6], based on shared tree, proposed an distributed algorithm that core does not need global knowledge of network topology. Instead, core only maintains the information about agents (sub-tree's root) and the information of all the members is distributed among local agents.

\section{Proposed Algorithm}

The network is modeled as a simple, undirected, and connected graph $G=$ $(V, E)$, where $V$ is the set of nodes and $E$ is the set of edges (or links). The nodes represent the designated routers and the edges represent the network communication links connecting the routers. An edge $e \in E$ connecting two adjacent nodes $u$ and $v$ will be denoted by $(u, v)$. Each edge $e$ has two non-negative metrics associated with it: a cost function $C(e)=C(u, v)$ represents the utilization of the link, and a delay function $D(e)=D(u, v)$ represents the delay that the packet experiences through passing that link including switching, queuing, transmission and propagation delays. A path $P\left(v_{0}, v_{n}\right)=\left(v_{0}, v_{1}, \cdots, v_{n}\right)$ in the network, has two associated characteristics: $C\left(P\left(v_{0}, v_{n}\right)\right)=\sum_{i=0}^{n-1} C\left(v_{i}, v_{i+1}\right)$ and $D\left(P\left(v_{0}, v_{n}\right)\right)=\sum_{i=0}^{n-1} D\left(v_{i}, v_{i+1}\right)$.

We denote $V_{T} \subset V$ as a set of routers to which hosts that belong to a multicast group are attached. For simplicity, we call the set $V_{T}$ a multicast group with each router $v \in V_{T}$ as a group member $1 \mathrm{~A}$ multicast tree $T=\left(V_{T}, E_{T}\right)$, which is a subgraph of $G$ that spans all the nodes in $V_{T}$ and has no cycles, has an associated cost defined as $C(T)=\sum_{e \in E_{T}} C(e)$.

Given a tree $T$ and two nodes $u$ and $v$ belonging to this tree, we will let $P_{T}(u, v)$ denote the path between $u$ and $v$ in this tree. Then, the delay and cost of this path are respectively denoted as $D\left(P_{T}(u, v)\right)$ and $C\left(P_{T}(u, v)\right)$. Let nodes $v_{i}$ and $v_{j}$ are the farthest and the nearest node away $u$, respectively, where $u$,

\footnotetext{
${ }^{1}$ Actually the multicast group should be the set of hosts that are directly attached to routers in $V_{T}$.
} 
$v_{i}$, and $v_{j}$ are all in $V_{T}$. We denoted by $\delta(u)=D\left(P_{T}\left(u, v_{i}\right)\right)-D\left(P_{T}\left(u, v_{j}\right)\right)$ the inter-destination delay variation of the node $u$.

Let $R=\left\{r_{1}, r_{2}, \ldots, r_{k}\right\}$ be a sequence of requests, where $r_{i}$ is either adding or removing a destination node to or from the multicast group. Let $V_{T_{i}}$ be the set of nodes in the multicast group after request $r_{i}$ has been completely made. In response to $r_{i}$, we also let $T_{i}$ and $u_{i} \in V_{T_{i}}$ be the multicast tree spanning $V_{T_{i}}$ and the core of the tree $T_{i}$, respectively.

There are two parameters are defined to characterize the quality of the tree as perceived by application performing the multicast. These parameters relate the end-to-end delays along individual core-destination paths to the desired level of QoS.

- Delay tolerance, $\Delta$ : Parameter $\Delta$ represents an upper bound on the acceptable end-to-end delay along any path from the core to a destination node. This parameter reflects the fact that the information carried by multicast packets become stale $\Delta$ time units after its transmission at the core.

- Inter-destination delay variation tolerance, $\delta(u)$ : This is the maximum allowed difference in end-to-end delay between the paths from the node $u$ (core) to its farthest and nearest nodes which are in the set $V_{T}$. In essence, this parameter is used to insure that no one is left behind and that none is far ahead in receiving the same data among the various receivers.

Problem 1. Given a network $G=(V, E)$, a delay tolerance $\Delta$, a shared tree $T_{0}$, rooted at $u_{0}$, which is a subgraph of $G$, and a request sequence $R=$ $\left\{r_{1}, r_{2}, \ldots, r_{k}\right\}$. With respect to request $r_{i}$ been made, the sequence of multicast tree $\left\{T_{1}, T_{2}, \ldots, T_{k}\right\}$ is constructed, in which $T_{i}$ spans $V_{T_{i}}$. After tree $T_{i}$ constructed, find a node $u_{i} \in V_{T_{i}}$ satisfies the following conditions:

1. Tree $T_{i}$ has minimum inter-destination delay variation. Formally, $\left\{u_{i} \in V_{T_{i}} \mid \delta\left(u_{i}\right)=\min _{v_{j} \in V_{T_{i}}} \delta\left(v_{j}\right)\right\}$.

2. The delay of path $P_{T_{i}}\left(u_{i}, v\right)$ fulfills the delay tolerance $\Delta$. That is, $D\left(P_{T_{i}}\left(u_{i}, v\right)\right) \leq \Delta, \quad \forall v \in V_{T_{i}}$.

3. After the above two conditions have been satisfied, the tree $T_{i}$ has minimum cost.

This problem is known to be NP-Complete since it reduces to the standard Steiner tree problem [57].

Please refer to Appendix A for detailed description of our approach and we briefly describe it in the rest of this section. We assume that a shared tree $T$, rooted at node $u$ (core), has been constructed at the beginning. The nodes that those are already on-tree nodes maintain their own table $T D$.The fields of $T D$ are Branch indicated the branch connecting to neighboring node and AccumulatedDelay indicated the accumulated delay of the farthest leaf node along Branch. We also assume that each node in network knows the delays that messages pass through adjacent branches to neighboring nodes. While GRAFT message is sent to an on-tree node $v_{j}$, it calculates the accumulated delay along the joining path, and decides whether to send an UPDATE message to upstream node of itself or not. 
The PRUNE message is only sent by leaf nodes, and is terminated at a node which has the other branch in $T D$ or is a member, too. When node $v_{j}$ receives a PRUNE message, it determines its own table $T D$ to verify whether maximum delay is replaced, then an UPDATE message is consequently sent to its upstream node. On the other hand, a PRUNE message is sent to upstream node of $v_{j}$, if there is no any record in $T D$.

According to the new arrival UPDATE message, a node $v_{j}$ may update its corresponding recode in $T D$. If the maximal AccumulatedDelay is changed and $v_{j}$ is not the core, then an UPDATE message is sent to upstream node, again. The above process is continued until the core $u$ receiving an UPDATE message. The core calculates the difference between first and second maximal accumulated delay in $T D$, and it decides whether migration process is proceed or not. If migration is necessary for best quality requirements, the core $u$ sends MIGRATE message along the branch toward the farthest node. However, each node which receives MIGRATE message is just a candidate of core. The candidate also calculates the next node along maximal delay path until no more better results. Consequently, the candidate core becomes the new core and sends a NOTIFY message to all the source nodes of multicast tree $T$.

\section{Simulation Model and Results}

In generating random graphs, we adopt the method used by Waxman [4, where vertices are placed randomly in a rectangular coordinate grid by generating uniformly distributed values for their $x$ and $y$ coordinates. The probability function $P(u, v)=\beta e^{\frac{-d(u, v)}{2 \alpha n}}$ and a random number $0 \leq r<1$ are used to decide that whether an edge exists between node $u$ and $v$ or not. Where $d(u, v)$ is denoted as their Euclidean distance, $\alpha$ and $\beta$ are tunable parameters, and $n$ is the number of nodes in the graph. Increasing $\alpha$ increases the number of connections between far off nodes and increasing $\beta$ increases the degree of each node.

To generate addition or deletion requests for our simulation, we have used the probabilistic model employed in [4]. In a network of size $N$, let $k$ represent the number of nodes in the multicast tree. Then, the probability of an add-request is given by the expression $\operatorname{Prob}(a d d)=\frac{\gamma(N-k)}{\gamma(N-k)+(1-\gamma) k}$, where $\gamma$ is a constant in the range $(0,1]$. The value of $\gamma$ determines the equilibrium point at which the probability of an add or delete request is equally likely. When $\gamma=\frac{k}{N}$, the above expression takes a value of 0.5 . The probability that a request will be a delete-request is given by $\operatorname{Prob}(\mathrm{del})=1-\operatorname{Prob}(a d d)$.

Our simulation studies were conducted on a set of 100 random networks. Values of $\alpha=0.2$ and $\beta=0.2$ were used to generate networks with average degree ranging between 3 and 4 . Each random network received a total of 30 update requests. These requests were generated based on the probabilistic model using a value of $\gamma=0.3$. Two performance measures, namely maximum inter-destination variation and cost of multicast tree, are used to evaluate the performance of the algorithms. The performance of the algorithms are also evaluated over two parameters, which are: number of network nodes and multicast group size. 


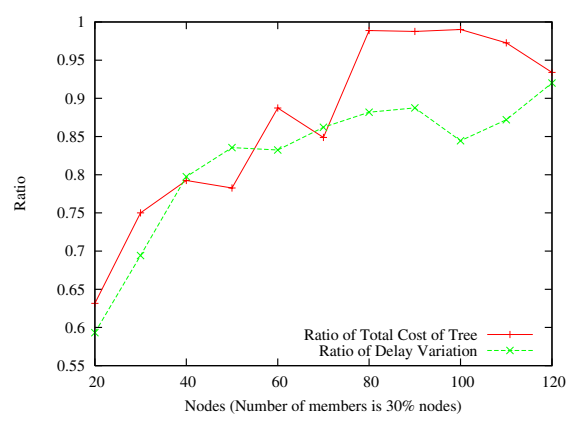

(a) Different number of nodes (group size 30\%)

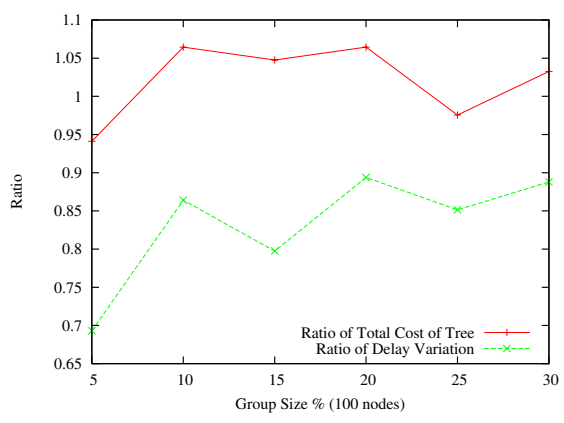

(b) Different number of group size (nodes 100)

Fig. 1. Performance comparisons for inter-destination delay variation

Fig. 1(a) shows the results for different number of network nodes, which ranges from 20 to 120 in steps 10 while group size are kept constant at $30 \%$ of network nodes. Fig. 1(b) shows the results for different number of group size, ranging from 5 to 30 in steps 5 while network size are kept constant at 100. It is easy to see that our algorithm is able to generate solutions with smaller inter-destination delay variation than SPT's algorithm.

\section{Conclusions}

In this paper, we provide the solution to multicast trees construction with the guarantees that the end-to-end delays from the core to the destination modes and the variation among these delays are within a given bound constrain. And our resolution is able to minimize total tree costs as well. The problem of constructing such trees is NP-complete. In comparing with SPT's algorithm, our heuristic exhibits good average case behavior, eases of implement, and does not need global network information during tree construction. Our heuristic does not take end-to-end delay bounds into account since satisfaction of the interdestination delay variation is the most important consideration in our strategy. However, our heuristic is flexible in modification to cope with the end-to-end delay bound requirement in many ways such as the method proposed in Rouskas's and Baldine's research [5]. The analysis of data losing rate during the core migration process should be explored more in the future research.

\section{References}

1. Ballardie, T., Francis, P., Crowcroft, J.: Core-based trees (CBT): An architecture for scalable inter-domain multicast routing. ACM SIGCOMM Computer Communication Review 23 (1993) 85-95

2. Donahoo, M.J., Zegura, E.W.: Core migration for dynamic multicast routing. In: Proceedings of the Fifth International Conference on Computer Communications and Networks (ICCCN '96). (1996) 92-98 
3. Carlberg, K., Crowsroft, J.: Building shared trees using a one-to-many joining mechanism. ACM SIGCOMM Computer Communication Review 27 (1997) 5-11

4. Waxman, B.M.: Routing of multipoint connections. IEEE Journal of Selected Areas in Communications 6 (1988) 1617-1622

5. Rouskas, G.N., Baldine, I.: Multicast routing with end-to-end delay and delay variation constraints. IEEE Journal of Selected Areas in Communications 15 (1997) 346-356

6. Wang, T.Y., Wuu, L.C., Huang, S.T.: A scable core migration protocol for dynamic multicast tree. Journal of Information Science and Engineering 19 (2003) 479-501

7. Kompella, V., Pasquale, J., Polyzos, G.: Multicasting for Multimedia Applications. In: Proc. IEEE INFOCOM '92, Florence, Italy (1992) 2078-2085

\section{Appendix A}

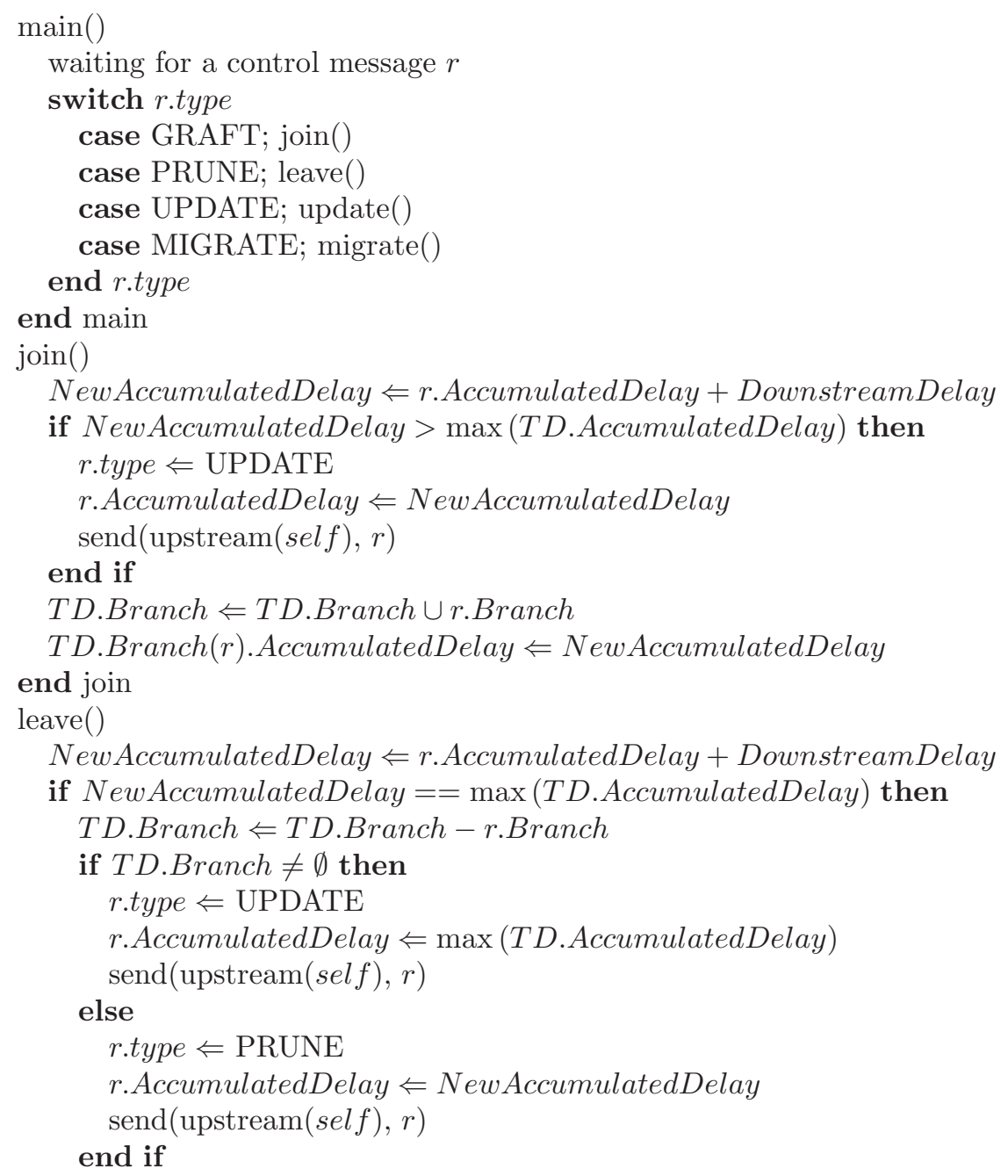


end if

end leave

update ()

NewAccumulatedDelay $\Leftarrow$ r.AccumulatedDelay + DownstreamDelay

OldMaxDelay $\Leftarrow \max (T D$.AccumulatedDelay $)$

if NewAccumulatedDelay $>$ TD.Branch $(r)$. AccumulatedDelay then

TD.Branch $(r)$.AccumulatedDelay $\Leftarrow$ NewAccumulatedDelay

end if

if OldMaxDelay $<\max (T D$. AccumulatedDelay) then

if self $\neq$ core then

r.type $\Leftarrow$ UPDATE

r.AccumulatedDelay $\Leftarrow \max (T D$.AccumulatedDelay $)$

send(upstream $($ self $), r$ )

else

MaxDelay $\Leftarrow \max (T D$. AccumulatedDelay $)$

SecMaxDelay $\Leftarrow$ secondly maximum in TD.AccumulatedDelay

BranchDelay $\Leftarrow$ the delay of branch toward farthest member

if (MaxDelay - SecMaxDelay)/ $2>$ BranchDelay then

r.type $\Leftarrow$ MIGRATE

r.AccumulatedDelay $\Leftarrow$ SecMaxDelay

send(maxdownstream $($ self $), r$ )

end if

end if

end if

end update

migrate()

NewAccumulatedDelay $\Leftarrow$ r.AccumulatedDelay + UpstreamDelay

TD.Branch $(r)$.AccumulatedDelay $\Leftarrow$ NewAccumulatedDelay

MaxDelay $\Leftarrow \max (T D$.AccumulatedDelay $)$

SecMaxDelay $\Leftarrow$ secondly maximum in TD.AccumulatedDelay

BranchDelay $\Leftarrow$ the delay of branch toward farthest member

if (MaxDelay - SecMaxDelay) / $2>$ BranchDelay then

r.type $\Leftarrow$ MIGRATE

r.AccumulatedDelay $\Leftarrow$ SecMaxDelay

send(maxdownstream $($ sel $f), r$ )

else

r.type $\Leftarrow$ NOTIFY

$\operatorname{send}(\operatorname{source}(T), r)$

end if

end migrate 